\title{
Contribuciones de la Medicina Psicosomática a la Medicina Clínica y Preventiva
}

\author{
Carmen Berrocal ${ }^{1 *}$, Giovanni A. Fava ${ }^{2}$ y Nicoletta Sonino ${ }^{3}$ \\ ${ }^{1}$ Departamento de Patología Quirúrgica, Médica, Moleculary del Área Crítica, Universidad de Pisa (Italia). \\ ${ }^{2}$ Departamento de Psicologia, Universidad de Bologna (Italia). \\ ${ }^{3}$ Departamento de Ciencias Estadisticas, Universidad de Padova (Italia).
}

Resumen: El trabajo ofrece una revisión actualizada de las principales con-
tribuciones de la Medicina Psicosomática a la medicina clínica y preventiva.
Su contenido se basa en los resultados de revisiones sistemáticas, meta-
análisis y estudios controlados. La evidencia acumulada indica que la pers-
pectiva psicosomática podría contribuir significativamente a mejorar los re-
sultados de la atención sanitaria, mediante una práctica clínica holística, la
evaluación de los factores psicosociales implicados en la vulnerabilidad in-
dividual y la integración de intervenciones psicológicas en medicina. Dicha
evidencia constituye el terreno ideal para afrontar necesidades emergentes
en sanidad, como la gestión de enfermedades crónicas y de síntomas inex-
plicables, el paciente como productor activo de salud, la prevención tem-
prana y una valoración de resultados que supere el reduccionismo biomédi-
co.
Palabras clave: medicina psicosomática; biopsicosocial; conducta de enPalabras clave: medicina
fermedad; atención sanitaria.

\section{Introducción}

El término "psicosomático" se ha usado, con frecuencia, para hacer referencia a fenómenos distintos, denotando o generando así cierta ambigüedad en su significado. A veces se ha usado para aludir a un movimiento renovador en Medicina; otras, para referirse a la investigación sobre factores psicológicos implicados en la etiología de las enfermedades físicas, para clasificar enfermedades en las que se hipotiza que los factores psicológicos jueguen un papel crucial o, incluso, como etiqueta genérica para denominar todos aquellos síntomas somáticos para los que no se dispone de una explicación médica demostrada. Ninguna de estas acepciones es, sin embargo, completa o, al menos, ninguna refleja el concepto de Medicina Psicosomática (MP) que se ha desarrollado en los últimos años (Fava \& Sonino, 2010). La ambigüedad del término "psicosomático" deriva, probablemente, de la identificación de la MP con algunos de los marcos teóricos y/o métodos de investigación particulares de entre los que han formado parte de su evolución histórica, lo cual ha dado lugar a importantes malentendidos sobre su definición y sus objetivos.

Heinroth (1818) introdujo el término "psicosomático", si bien la moderna MP se desarrolló durante la primera parte del s. XX como resultado de la confluencia de dos conceptos que tienen una antigua tradición en la Medicina y en el pensamiento occidental: el de psicogénesis y el de holismo (Lipowski, 1986). El primero caracterizó ampliamente la primera fase de desarrollo de la MP (1930-1960) y desembocó en el concepto de "trastorno psicosomático" -

* Dirección para correspondencia [Correspondence address]:

Carmen Berrocal Montiel. DIPINT, via Savi 10, 56126 Pisa (Italia).

E-mail: carmen.berrocalmontiel@med.unipi.it
Title: Contributions of Psychosomatic Medicine to Clinical and Preventive Medicine.

Abstract: This paper provides an updated review of the main contributions of Psychosomatic Medicine to clinical and preventive medicine. Metaanalyses, comprehensive reviews and controlled studies were selected. Current advances in the field indicate that health care can be improved by using a psychosomatic approach that takes into account psychosocial factors affecting individual vulnerability, the holistic consideration of patient care, and the integration of psychological interventions in medicine. Consolidated evidence in Psychosomatic Medicine provides the ideal background for dealing with new needs emerging in medical practice, including chronic illness management, somatic unexplained symptoms, the shift in the role of patients from health consumers to actively health producers, primary earlylife prevention, and the appraisal of health outcomes beyond the biomedical reductionism.

Keywords: psychosomatic medicine; biopsychosocial; illness behavior; health care.

enfermedad cuya causa es atribuida a factores psicológicos. A pesar de las críticas iniciales (Halliday, 1948), el postulado psicogénico ejerció una considerable atracción, particularmente en el seno del Psicoanálisis. Así, mediante la observación clínica propia del Psicoanálisis y de la Psicología Dinámica posterior, varias enfermedades de origen desconocido recibieron la denominación de "trastornos psicosomáticos" para enfatizar con ello una (hipotética) patogénesis intrapsíquica.

La evolución ulterior de la MP ha estado marcada por las contribuciones, entre otros, de Engel, Lipowski y Kissen, quienes asentaron, durante los años 60, las bases de la actual MP. Engel (1967) propuso un modelo multifactorial, "biopsicosocial" (Engel, 1977), en el que la enfermedad es concebida como resultado de la continua interacción de factores biológicos, psicológicos y sociales. Así, el estudio de la enfermedad debería incluir el individuo, el organismo y el ambiente como componentes esenciales del sistema como un todo (Engel, 1977; Fava, Ruino, Romba, \& Wise, 2012; Novack et al., 2007), en lo que Hinkle (1967) definió “perspectiva ecológica". Desde esta perspectiva, no es que algunas enfermedades sean psicosomáticas y otras no, sino que todos los procesos de enfermedad $-\mathrm{y}$ en sus distintas fasesderivan de la interacción de factores biopsicosociales. En el trabajo de Engel también emerge la orientación científica de la investigación psicosomática combinando métodos y técnicas fisiológicas y psicológicas.

Lipowski (1986) delimitó el ámbito de trabajo, los objetivos y métodos de la MP. Además, criticó la noción obsoleta de psicogénesis, incompatible con el concepto de multicausalidad. De hecho, la MP ha abandonado el concepto de psicogénesis a lo largo de su travesía histórica para adoptar una concepción multifactorial de la enfermedad como postulado 
central. Por su parte, Kissen (1963) enfatizó que el peso de los factores psicosociales puede variar de un individuo a otro dentro de la misma enfermedad, destacando así el error conceptual básico de considerar las enfermedades como entidades homogéneas.

Así, abrazando la unidad mente-cuerpo, y en contraste con una visión reduccionista de la enfermedad, el término psicosomático denota hoy una perspectiva clínica y metodológica desde la que se estudia el rol de los factores psicosociales sea en las enfermedades para las que no se cuenta con una patogénesis orgánica conocida, denominadas por ello "funcionales", sea en las enfermedades conocidas como "orgánicas".

La investigación psicosomática ha generado un notable cuerpo de conocimientos, objeto de numerosas publicaciones en las principales revistas médicas. Este trabajo ofrece una definición actualizada de la MP, esboza sus fronteras con otros sectores relacionados, ilustra sus principales contribuciones a la medicina clínica y preventiva y discute algunas de sus principales líneas de desarrollo futuro (Fava \& Sonino, 2010). La mayor parte del contenido se basa en revisiones sistemáticas de la literatura, meta-análisis y estudios controlados. En particular, se han seleccionado los artículos relevantes para la práctica clínica.

\section{Definición y fronteras con otras disciplinas}

De acuerdo con la definición original de Lipowski (1986) y sus desarrollos posteriores (Fava \& Sonino, 2000, 2005), la MP puede ser definida como un marco comprehensivo, interdisciplinar, para: a) la evaluación de factores psicosociales implicados en la vulnerabilidad individual, el curso y los resultados de cualquier tipo de enfermedad; b) la consideración holística de la asistencia clínica; y c) la integración de las intervenciones psicológicas en el campo de la prevención, tratamiento y rehabilitación de las enfermedades médicas.

El American Board of Medical Specialities ha reconocido la MP como sub-especialidad (Gitlin, Levenson \& Lyketsos, 2004), lo cual podría llevar a equipararla con la Psiquiatría de Enlace (Gitlin et al., 2004), una sub-especialidad de la Psiquiatría dedicada al diagnóstico, tratamiento y prevención de la morbilidad psiquiátrica en pacientes médicos mediante el asesoramiento psiquiátrico y la coordinación y formación de profesionales de la salud, particularmente en el contexto hospitalario (Lipowski, 1983). La Psiquiatría de Enlace se ubica dentro del ámbito de la Psiquiatría. Su contexto de acción es la clínica y se ocupa de las condiciones psiquiátricas comórbidas de pacientes con enfermedades médicas (Wise, 2000). La MP es, por definición, multidisciplinar (Fava \& Sonino, 2000, 2005; Lipowski, 1986). No se limita al campo de la Psiquiatría, sino que interesa a cualquier otro sector médico. De hecho, es precisamente por ello que las actividades de la MP han alcanzado un estatus independiente en países como Alemania o Japón y, a menudo, se encuentran estrechamente relacionadas con la Medicina Interna (Deter, 2004).
Por otro lado, hasta los años 70 del s. XX, la MP era el único sector de investigación que servía de puente entre la Medicina y las ciencias comportamentales. Sin embargo, durante dichos años tuvo lugar el desarrollo de la Medicina Conductual (Schwartz \& Weiss, 1978), un campo interdisciplinar cuyo objetivo principal es el desarrollo y la integración de los conocimientos y técnicas biomédicas y comportamentales relevantes para la salud y la enfermedad. La Medicina Conductual ha impulsado considerablemente la investigación básica sobre cuestiones con notables implicaciones clínicas, como los mediadores biológicos de la relación entre determinadas variables psicológicas y algunos procesos de enfermedad (Miller, 1983).

$\mathrm{El}$ interés de la Medicina Conductual en las conductas de salud y los factores de riesgo que involucran el comportamiento contribuyó al nacimiento de la Psicología de la Salud (Hafen, Jarren, Frandsen, \& Smith, 1996). Mientras la MP es un campo interdisciplinar, la Psicología de la Salud es una branca específica de la Psicología y, como tal, se ocupa de las contribuciones de la Psicología para "la promoción y mantenimiento de la salud, la prevención y tratamiento de la enfermedad, la identificación de correlatos etiológicos y diagnósticos de la salud, de la enfermedad y de las disfunciones afines, y para el análisis y mejora del sistema sanitario, así como para la configuración de las políticas sanitarias" (Matarazzo, 1980, p. 815).

\section{Factores psicosociales que influyen en la vul- nerabilidad individual}

La atención clínica mejoraría significativamente si la evaluación médica incluyera variables de carácter psicosocial, en particular el estrés (Fava \& Sonino, 2000; Kissen, 1963; McEwen, 2007; Novack et al., 2007), pero también otros muchos factores psicosociales implicados en la vulnerabilidad a la enfermedad (Kroenke, 2002). Algunos factores, como las conductas de salud o el bienestar psicológico, no sólo se asocian a la prevención o a la mejora de la enfermedad sino que, además, promueven la salud (Ruini \& Fava, 2013).

Estresores tempranos. El efecto de los estresores experimentados durante las primeras fases de desarrollo evolutivo sobre la vulnerabilidad a la enfermedad ha sido objeto de numerosos estudios (Faravelli et al., 2012; McEwen, 2007; Novack et al., 2007). Mediante el uso de análogos animales, se ha observado que eventos como la separación materna temprana se asocian, consistentemente, con el desarrollo de modificaciones patofisiológicas como, por ejemplo, una mayor activación del eje hipotalámico-pituitario-adrenal (HPA) (McEwen, 2007; Novack et al., 2007). De esta forma, los estresores tempranos pueden incrementar la vulnerabilidad del individuo a los efectos del estrés en un momento posterior de la vida.

Por otra parte, el maltrato infantil se asocia a un mayor uso de los servicios de salud durante la etapa adulta (Applebaum, Nemets, Kaplan, Witztum, \& Belmaker, 2012; 
Arnow, 2004; Bonomi et al., 2008). Algunos estudios también sugieren que se relaciona con un mayor nivel de discapacidad funcional y de conductas de riesgo para la salud (Walker et al., 1999), aunque la evidencia disponible no es consistente (Romans \& Cohen, 2008). La relación entre el maltrato infantil y condiciones como el dolor crónico o el síndrome del intestino irritable ha sido también objeto de considerable interés (McCauley et al., 1997).

Estrés y carga alostática. La adopción de métodos de investigación rigurosos ha permitido corroborar la asociación entre los estresores vitales y numerosas enfermedades médicas - endocrinas, cardiovasculares, respiratorias, gastrointestinales, inmunológicas, oncológicas y dermatológicas (McEwen, 2007; McEwen \& Stellar, 1993; Picardi \& Abeni, 2001; Rozanski, Blumenthal, \& Kaplan, 1999; Sonino, Tomba, \& Fava, 2007; Wright, Rodríguez, \& Cohen, 1998). Asímismo, los eventos "menores" pueden constituir una importante fuente de estrés crónico cotidiano. Mc Ewen y Stellar (1993) han formulado la relación estrés-enfermedad en términos de "alostasis", destacando la habilidad del organismo para obtener estabilidad a través del cambio. Mediante la alostasis, el SNA, el eje HPA y los sistemas cardiovascular, metabólico e inmunológico protegen el organismo respondiendo al estrés interno y externo.

La carga alostática hace referencia al desgaste y deterioro que se produce como consecuencia de un estrés excesivo o de un afrontamiento que resulta insuficiente o inadecuado para reducir la respuesta de estrés cuando ésta ya no es necesaria. Distintos indicadores biológicos de la carga alostática como las proteínas glicosiladas o marcadores hormonales y de coagulación/fibrinolisis - se asocian con un peor funcionamiento físico y cognitivo y con mayores tasas de mortalidad (McEwen, 2007). Los parámetros biológicos de carga alostática presentan, sin embargo, diversas limitaciones. Además de no ofrecer una visión global del estado de desequilibrio del organismo, se trata de mediadores inespecíficos que pueden ser influenciados por variables diversas de un individuo a otro (Fava, Guidi, Semprini, Tomba, \& Sonino, 2010).

Recientemente se han propuesto diversos criterios clínicos -basados en los eventos de estrés (reciente y crónico) y en el distrés psicológico- para obtener un índice clinimétrico de la carga alostática (Fava et al., 2010). El índice clinimétrico correlaciona negativamente con el funcionamiento psicosocial y positivamente con psicopatología, desgaste emocional y conductas de enfermedad en pacientes con enfermedades cardiovasculares crónicas (Porcelli, Domenico, Domenico, \& Di Masi, 2012). Igualmente, correlaciona positiva y significativamente con ansiedad y depresión y negativamente con bienestar psicológico en población general (Tomba \& Offidani, 2012). Merecería la pena explorar la validez de dicho índice para discriminar patrones neuroendocrinos significativos y su utilidad para prevenir o reducir el impacto del estrés sobre la salud (Fava et al., 2010).

Conductas de salud. Entre los principales factores de riesgo de muchas de las enfermedades de mayor prevalencia en los países desarrollados (como la diabetes o las enfermedades cardiovasculares) destacan los estilos de vida poco saludables (Mokdad, Marks, Stroup \& Gerberding, 1998). Ya en el 1985, Rose mostró que los factores de riesgo para la salud casi siempre siguen una distribución normal y defendió un enfoque a la prevención focalizado sobre la población general, en vez de orientado hacia los grupos de riesgo como es habitual. Recientemente se ha enfatizado de nuevo la necesidad de renovar las prácticas en atención primaria incorporando la modificación de las conductas de salud (Aspy et al., 2008; Green, Cifuentes, Glasgow, \& Stange, 2008). En la misma línea, la American Academy of Pediatrics (2008) ha destacado la necesidad de abordar la "epidemia" de obesidad que afecta a la infancia mediante programas dirigidos a modificar los hábitos de alimentación y de actividad física (Daniels \& Greer, 2008).

Apoyo social. Distintos estudios prospectivos en población general revelan asociaciones significativas entre diversas medidas de apoyo social e indicadores de salud, como el nivel de adaptación a, y recuperación de, las enfermedades crónicas, o las tasas de mortalidad y morbilidad, psiquiátrica y física (Fava \& Sonino, 2005). Un bajo apoyo social es, además, un predictor significativo de un mayor uso de los servicios de salud (Vedsted \& Christensen, 2005). Existen, por otra parte, intervenciones psicosociales, orientadas a la modificación del ambiente social y las relaciones interpersonales, que favorecen la adaptación del individuo a la enfermedad (Cohen \& Gottlieb, 2000).

Por su parte, la denominada "neurociencia social" ha iniciado a abordar los efectos del ambiente social sobre el cerebro y sobre distintas funciones fisiológicas reguladas a nivel central (McEwen, 2007). Como han sugerido algunos autores (Seeman, Singer, Ryff, Dienberg, \& Levy-Storms, 2002), el apoyo social parece ser un potente factor de protección de la salud, sobre todo en relación al proceso de estrés, dado que disponer de relaciones sociales positivas se asocia a una menor carga alostática.

Bienestar psicológico. Con frecuencia, la salud se equipara a ausencia de enfermedad a pesar de que, hace ya más de medio siglo, la OMS definió la salud como un "estado de completo bienestar físico, mental y social, y no solamente la ausencia de afecciones o enfermedades" (WHO, 1948). El bienestar psicológico ha sido operativizado mediante varias dimensiones interrelacionadas, cuales el "control ambiental", "crecimiento personal", "propósito en la vida", "autonomía", "auto-aceptación" y "relaciones sociales positivas" (Ryff \& Singer, 1996). Algunos estudios sugieren que el bienestar amortigua el impacto negativo del estrés y, como consecuencia, tiene un efecto favorable sobre el curso de la enfermedad (Chida \& Steptoe, 2008; Cosci, Pennato, Bernini, \& Berrocal, 2011; Pressman \& Cohen, 2005).

Un concepto relacionado, concebido en un marco salutogénico, es el "sentido de coherencia" de Antonovsky (Eriksson \& Lindstrom, 2006), el cual remite a los recursos que facilitan la gestión del estrés, a la reflexión sobre los propios recursos para afrontar los desafíos de la vida y al uso 
de estrategias de coping eficaces. El "sentido de coherencia" se asocia con una mejor salud percibida, especialmente mental, y es un importante factor involucrado en el mantenimiento de la salud (Eriksson \& Lindstrom, 2006).

Personalidad. La consideración de la personalidad como factor de vulnerabilidad de distintas enfermedades fue una hipótesis frecuente durante la primera etapa de la MP, sobre todo en el seno del Psicoanálisis. Las propuestas de perfiles de personalidad específicos subyacentes a ciertas "enfermedades psicosomáticas" no han recibido, sin embargo, apoyo empírico (Hafen et al., 1996; Lipowski, 1986).

El patrón de comportamiento tipo A y la alexitimia parecen ejercer un efecto sobre la vulnerabilidad general a la enfermedad, si bien la relación de ambas variables con indicadores de salud es controvertida (De Vries, Forni, Voellinger \& Stiefel, 2012; Grabe et al., 2008; Shan, White, White, Littler, \& 2004; Taylor \& Bagby, 2004). Asímismo, el estudio Gubenberg indica que el patrón de comportamiento tipo D, muy relacionado con la alexitimia (Ogrodniczuk, Sierra, Sochting, Joyce, \& Piper, 2012), es un factor de riesgo independiente de la enfermedad coronaria y se asocia significativamente con altos niveles de depresión y ansiedad, peor estado de salud, mental y físico, y mayor uso de los servicios sanitarios (Beutel et al., 2012).

Por otra parte, el modelo socio-cognitivo presupone que las variables de personalidad interaccionan con factores ambientales y sociales dando lugar a importantes diferencias en las características de las situaciones que la persona selecciona (Mischel \& Shoda, 1995). Así, las variables de personalidad pueden ejercer un amplio efecto sobre el tipo y la cantidad de eventos de estrés que la persona tendrá que afrontar y sobre la representación cognitiva de diversas dimensiones de la enfermedad (p.ej., las causas, el tratamiento, etc.) así como de sus interacciones sociales, incluídas las relaciones con el personal sanitario.

\section{Consideración holística de la atención al pa- ciente}

Los factores psicosociales y biológicos interaccionan de muy distintas formas durante el curso de una enfermedad. La diversa influencia que dichos factores ejercen contribuye a determinar la cualidad de la experiencia y la actitud única de cada paciente en cada episodio de enfermedad.

Trastornos mentales. La relación entre los trastornos mentales -en particular, ansiedad y depresión- y las enfermedades médicas es estrecha (Katon, 2003). Los primeros incrementan el riesgo de sufrir enfermedades físicas y viceversa, y dicha comorbilidad complica sea la identificación que el tratamiento del trastorno médico (Prince et al., 2007). La relación entre ambos tipos de trastornos puede variar desde una relación puramente casual hasta una relación causal en la que los factores orgánicos -la alteración orgánica y/o el tratamiento farmacológico- causan el trastorno mental. Estos últimos casos se suelen denominar "trastornos mentales or- gánicos", en los que los síntomas psiquiátricos remiten tras el tratamiento de la alteración orgánica (Lishman, 1998), como la depresión en el síndrome de Cushing (Sonino et al., 2007).

El diagnóstico correcto de depresión en atención primaria no es fácil. De hecho, un reciente meta-análisis indica que son más los falsos positivos que los falsos negativos o que los casos correctamente identificados (Mitchell, Vaze, \& Rao, 2009). En cualquier caso, la depresión mayor ejerce un efecto negativo sobre la calidad de vida, el funcionamiento social del individuo y la adherencia terapéutica; se asocia a un mayor uso de los servicios de salud y a tasas de mortalidad más elevadas, particularmente durante la vejez (Di Matteo, Lepper, \& Croghan, 2000; Frasure-Smith \& Lesperance, 2003; Katon, 2003; Schulz, Drayer, \& Rollman, 2002). La investigación ha puesto igualmente en evidencia diversas implicaciones clínicas negativas de la comorbilidad entre las enfermedades físicas y los trastornos de ansiedad (Roy-Byrne et al., 2008).

Factores psicológicos. La Psiquiatría enfatiza la evaluación de los signos y síntomas de los distintos síndromes propuestos en las clasificaciones al uso (DSM). Sin embargo, la evidencia muestra que existen síntomas subclínicos y procesos psicológicos no encuadrables en las categorías diagnósticas que influyen en la calidad de vida y tienen implicaciones patofisiológicas y terapéuticas relevantes (Fava, 1999a). Lo anterior ha llevado, de hecho, al desarrollo de los Diagnostic Criteria for Psychosomatic Research (DCPR) (Fava, Fabbri, Sirri, \& Wise, 2007; Porcelli \& Sonino, 2007), los cuales han sido validados en diversos contextos médicos (Fava et al., 2007; Ferrari, Galeazzi, Mackinnon, \& Rigatelli, 2008; Porcelli \& Sonino, 2007; Porcelli et al., 2009; Venditti, Cosci, Bernini, \& Berrocal, 2013).

Los DCPR permiten identificar distintos factores psicológicos (desmoralización, irritabilidad, comportamiento tipo A, alexitimia) que ejercen un impacto sobre las enfermedades físicas (Sirri \& Fava, 2013). Además, proporcionan una clasificación de las conductas de enfermedad (ansiedad por la salud, negación de la enfermedad, fobia a la muerte y a la enfermedad, somatización, síntomas de conversión, síntomas somáticos funcionales, reacción de aniversario), las cuales reflejan distintos modos mediante los cuales las personas perciben, experimentan, evalúan y responden al propio estado de salud (Sirri, Grandi, \& Fava, 2008). La conducta de enfermedad es un predictor significativo del uso de los servicios sanitarios, la automedicación y la adherencia terapéutica, ejerciendo así un impacto nada desdeñable sobre los costes médicos y la carga laboral del personal sanitario (Sirri, Fava, \& Sonino, 2013). De hecho, la conducta de enfermedad ha sido propuesta como un concepto unificador que podría explicar parte de la variabilidad de la respuesta terapéutica y el pronóstico de pacientes que sufren la misma enfermedad (Sirri et al, 2013).

La evaluación diagnóstica mediante el DSM ha sido criticada por su limitada utilidad clínica, y la nueva edición de dichos criterios continúa adoleciendo de las mismas debilida- 
des (Sirri \& Fava, 2013). En particular, los criterios DSM-5 (APA, 2013) para el diagnóstico de los trastornos con síntomas somáticos descuidan importantes conductas de enfermedad (p.e., la negación de la enfermedad) que ejercen un impacto sobre las condiciones médicas, proporcionando así una representación incompleta o reducida de la respuesta del paciente a los síntomas físicos y a la enfermedad. Los DCPR capturan variables psicológicas que no son contempladas en las clasificaciones psiquiátricas, enriqueciendo de este modo el espectro de información disponible para el proceso de decisión clínica (Sirri \& Fava, 2013).

Calidad de vida. En tanto no existe ni una definición precisa de calidad de vida ni consenso sobre cuál debería ser ésta, la investigación en este campo ha explorado, particularmente, el funcionamiento cotidiano del individuo y la percepción de salud del paciente (Testa \& Simonson, 1996). En diversas condiciones médicas como, por ejemplo, el dolor crónico, el nivel de funcionamiento cotidiano del paciente depende, no tanto de variables médicas como la severidad o la duración de la enfermedad, sino de variables de naturaleza psicosocial (p.ej., la evitación experiencial) (Bernini, Pennato, Cosci, \& Berrocal, 2010).

Es más, como algunos autores han argumentado, los indicadores biomédicos no son suficientes, por sí solos, para evaluar adecuadamente los efectos de los procesos de enfermedad y de las intervenciones terapéuticas (De Fruyt \& Demyttenaere, 2009; Kaplan, 2003; Testa \& Simonson, 1996). De hecho, la inclusión de variables como el funcionamiento cotidiano o la calidad de vida podría llevar a conclusiones diferentes sobre la eficacia de ciertas intervenciones. Un tratamiento podría tener efectos positivos sobre los indicadores biológicos de la enfermedad y, sin embargo, no incidir sobre las expectativas de vida, el funcionamiento cotidiano o la calidad de vida del individuo; es más, un tratamiento, aun pudiendo tener efectos positivos sobre los indicadores biológicos de enfermedad, podría reducir las expectativas o la calidad de vida, lo cual cuestionaría seriamente su uso (Kaplan, 2003). La inclusión, por tanto, de la calidad de vida como medida de resultado es fundamental para poder llevar a cabo una adecuada valoración, en términos de costes-beneficios, de los procedimientos diagnósticos y de las intervenciones médicas.

\section{Integración de las intervenciones psicológicas en Medicina}

Diversos profesionales de la salud (psiquiatras, psicólogos, profesionales de enfermería, etc.) pueden implementar intervenciones psicológicas en el campo de la enfermedad médica. Dichas intervenciones pueden oscilar desde la mera comunicación afectiva y confortante hasta tratamientos psicológicos y psicofarmacológicos específicos. Muchos de los estudios iniciales sobre la eficacia de los tratamientos psicológicos se han llevado a cabo con especialistas entrenados y con experiencia. Sin embargo, un creciente número de estu- dios sugiere que clínicos sin formación específica en Psiquiatría o en Psicología pueden aplicar dichas intervenciones con eficacia (Moorey et al., 2009; Narayan, Kanaya, \& Gregg, 2003), ampliándose así la disponibilidad de recursos para afrontar de forma integrada fenómenos cruciales para la salud, desde el estilo de vida hasta las conductas de enfermedad.

Modificación del estilo de vida. Un cuerpo creciente de evidencia vincula la evolución de algunas enfermedades severas con conductas específicas del estilo de vida (Djoussé, Driver, \& Graziano, 2009; Forman, Stampfer, \& Curhan, 2009; Mokdad et al., 2004; Narayan et al., 2003; Stone, 2004). Los beneficios consiguientes a la modificación del estilo de vida son particularmente evidentes en las alteraciones coronarias (Rozanski et al., 1999) y en la diabetes tipo 2 (Narayan et al., 2003). En la misma línea, distintos tratamientos psicológicos se han demostrado eficaces para modificar comportamientos de riesgo como, por ejemplo, el tabaquismo (Compas, Haaga, Keefe, Leitenberg, \& Williams, 1998).

En este contexto, la "colaboración" del paciente en el proceso de gestión de la enfermedad, uno de los supuestos básicos de la MP, es fundamental. El paradigma de colaboración mutua se apoya en una relación médico-paciente en la que las decisiones se adoptan conjuntamente (Joosten, DeFuentes-Merillas, de Weert, Sensky, van der Staak, \& de Jong, 2008) así como en el uso de estrategias de autoregulación para incrementar la autoeficacia del paciente (Bodenheimer, Lorig, Holman, \& Grumbach, 2002). Éstas son particularmente eficaces en las enfermedades crónicas y en el área de la rehabilitación, donde el comportamiento del individuo es crucial para una adecuada gestión del problema (Leventhal, Weinman, Leventhal, Phillips, \& 2008; Sonino \& Fava, 2007).

Tratamiento de la comorbilidad psiquiátrica. El tratamiento de la comorbilidad psiquiátrica como la depresión, aunque no ejerza efectos sobre las variables biomédicas, no sólo reduce los síntomas depresivos sino que también incrementa el funcionamiento cotidiano y la calidad de vida del paciente (Jackson, de Zee, \& Berbano, 2004). En cualquier caso, el tratamiento del distrés psicológico se asocia a una reducción del uso de los servicios de salud y, consiguientemente, de los gastos sanitarios (Konnopka et al., 2012).

Intervenciones psicosociales. Diversos estudios muestran que ciertas estrategias psicológicas (p.ej., cognitivoconductuales, psicoterapia dinámica breve) producen una mejoría sustancial en varias alteraciones médicas (Abbass, Kisely, \& Kroenke, 2009; Kaupp, Rapaport-Hubschman, \& Spiegel, 2005; Leventhal et al., 2008; Sharpe \& Schrieber, 2012). Como meros ejemplos, cabe destacar los programas para mejorar el apoyo social, el estado del humor y las conductas de salud en pacientes con cáncer de mama (Andersen et al., 2008), a fomentar las habilidades de autocontrol y auto-regulación en el dolor crónico (Turk, Swanson, \& Tunks, 
2008) o a promover la expresión emocional (Frisina, Borod, \& Lepore, 2004).

La Psicología Positiva ha dado a la luz intervenciones para promover el bienestar psicológico que pueden ser integradas en el campo de la MP. Un ejemplo de ello es la Wellbeing Therapy (WBT; Fava, 1999b), una intervención breve basada en el modelo de bienestar psicológico de Ryff (1989). Estudios con jóvenes y adolescentes sugieren que, además de incrementar el bienestar, la WBT reduce el distrés psicológico (Ruini \& Fava, 2013). Dichos resultados son particularmente relevantes si se tiene en cuenta que el bienestar y la afectividad positiva parecen ser factores de protección de la salud (Chida \& Steptoe, 2008; Fava \& Sonino, 2010; Ryff \& Singer, 1996, 1998; Seeman et al., 2002), por lo que la WBT constituye una prometedora estrategia de prevención y promoción de la salud en población general (Ruini \& Fava, 2013).

Por otra parte, la investigación sobre los tratamientos psicológicos ha revelado algunos ingredientes de uso común y transversales a la mayor parte de estrategias psicoterapéuticas que pueden ser aplicados al interno de cualquier relación médico-paciente. Destacan, entre ellos, la disponibilidad del terapeuta durante ciertos momentos del proceso terapéutico; la posibilidad de ventilar pensamientos y sentimientos; una relación de confianza y con alta carga emocional con una persona que proporciona ayuda; una explicación plausible de los síntomas; y un procedimiento que requiere la participación activa del paciente y del terapeuta y que es percibido por parte de ambos como una vía para restablecer la salud (Fava \& Sonino, 2005).

Tratamiento de la conducta anormal de enfermedad. Durante muchos años, la conducta anormal de enfermedad ha sido concebida como expresión de factores predisponentes de personalidad y reacia a las intervenciones psicológicas. La evidencia acumulada permite hoy cuestionar dicha suposición (Fava \& Sonino, 2005; Fava et al., 2007; Porcelli \& Sonino, 2007). Por ejemplo, estudios controlados indican que la hipocondría puede ser modificada mediante técnicas cognitivas (Bouman \& Visser, 1998; Clark et al., 1998). Asímismo, existen programas educativos y cognitivoconductuales eficaces para modificar conductas de enfermedad como los síntomas somáticos funcionales (Konnopka et al., 2012). Además, la relación entre las conductas anormales de enfermedad y los hábitos de salud tiene importantes implicaciones para las campañas de prevención. Por ejemplo, se ha observado que las personas con excesiva ansiedad por la salud cuidan peor de sí mismas que sujetos control (Sirri et al., 2008).

\section{Conclusiones}

Las necesidades sanitarias han cambiado profundamente durante las últimas décadas. Las enfermedades crónicas se han convertido en la principal causa de discapacidad y, aproximadamente, el $80 \%$ de los gastos sanitarios proviene del uso de los servicios de salud (Sonino \& Fava, 2007). A pesar de ello, la asistencia continúa siendo conceptualizada en términos de atención aguda y percibida como un "proveedor de productos de consumo". Como ha enfatizado Hart (1995), cuando el "producto" es la salud, el paciente no es sólo "consumidor" sino también uno de los "productores". Consiguientemente, para que el proceso de producción de salud sea eficiente es necesario que tenga lugar un cambio general de perspectiva, en modo que el paciente abandone el papel pasivo y antagonista de "consumidor" de salud para convertirse, junto con los profesionales de la salud, en "productor activo de salud" (Hart, 1995).

La consideración del funcionamiento cotidiano, el desempeño de los roles sociales, la estabilidad emocional, la calidad de vida o el bienestar del paciente, ha emergido como una necesidad crucial en el ámbito de la investigación y en la asistencia clínica (Testa \& Simonson, 1996). Dichos aspectos adquieren una relevancia particular en las enfermedades crónicas, en las que la curación no es posible, y la atención a los mismos se extiende sea a los familiares y cuidadores de los enfermos crónicos sea a los propios profesionales de la salud. De hecho, los propios pacientes son cada vez más conscientes de la relevancia de dichos aspectos. El éxito comercial de textos sobre medicina alternativa y prácticas positivas de salud, así como la difusión de la medicina mente-cuerpo, ejemplifican la receptividad del público general a mensajes sobre la promoción de la salud y modelos médicos alternativos. Las intervenciones psicosomáticas podrían responder a estas necesidades emergentes y representar un apoyo fundamental en el proceso de curación.

Por otra parte, en más de un $30-40 \%$ de los pacientes médicos se observan síntomas físicos inexplicables que incrementan significativamente el uso de los servicios y los gastos sanitarios (Konnopka et al., 2012; Kroenke, 2002; Lipowski, 1986), con costes comparables a los derivados de los trastornos afectivos (Konnopka et al., 2012). Las especialidades médicas tradicionales, organizadas principalmente de acuerdo a los sistemas orgánicos (p.ej., cardiología, dermatología...), son cada vez más inadecuadas para abordar síntomas y problemas que trascienden las subdivisiones del organismo y que requieren una perspectiva holística. El carácter interdisciplinar de la mayor parte de las clínicas de rehabilitación y del dolor es, precisamente, una consecuencia práctica de dicha observación (Leventhal et al., 2008; Sonino \& Fava, 2007). La activación de centros de tratamiento psicológico en el sistema sanitario nacional del Reino Unido es una oportunidad sin precedentes para la integración de procedimientos terapéuticos diversos (Layard, 2006; Marks, 2009). De hecho, la evidencia sugiere que la integración en el contexto médico de intervenciones educativas y cognitivoconductuales para tratar diversos comportamientos de enfermedad como los síntomas somáticos funcionales contribuiría a reducir los costes sanitarios (Konnopka et al., 2012).

Por otra parte, cada vez es más cuestionable el incremento exponencial de los gastos de la medicina preventiva focalizada en una pequeña parte de la población, los grupos de riesgo (Heath, 2006). Paralelamente, cada vez son más evi- 
dentes los beneficios asociados a la modificación del estilo de vida de la población general (Compas et al., 1998; Leventhal et al., 2008; Narayan et al., 2003), en consonancia con el modelo biopsicosocial. En la misma línea, los beneficios para la salud de la población podrían ser mayores si se abordaran las desigualdades en la atención sanitaria en la infancia, en vez de concentrar la mayor parte de los recursos en programas para la modificación de conductas de salud o incrementar el acceso a los servicios durante la etapa adulta (Shonkoff, Boyce, \& McEwen, 2009).

Lo anterior requiere una renovación no sólo del concepto de enfermedad y de las prácticas terapéuticas sino también del proceso de evaluación clínica y de valoración de los resultados. En Medicina existe la tendencia a preferir "datos duros" -expresados estos, preferiblemente, mediante cifras procedentes de medidas de laboratorio-, y a excluir la información "light", como la referida a los problemas, disfunciones o bienestar del individuo. Sin embargo, si los análisis sobre los costes-beneficios de las intervenciones sanitarias tuvieran en cuenta dicha información "light", las conclusiones respecto a cuáles son los servicios que producen más salud, a menor coste, podrían ser diversas (Kaplan, 2003). Asímismo, dicha información es fundamental para un adecuado juicio clínico que tenga en cuenta la respuesta individual al proceso de enfermedad (Fava \& Sonino, 2009; Tomba \& Bech, 2012). En la actualidad, dicha información puede ser evaluada mediante escalas e índices válidos y fiables, desarrollados por la clinimetría en el contexto de la investigación y práctica psicosomática (Fava \& Sonino, 2009; Tomba \& Bech, 2012).

\section{Referencias}

Abbass, A., Kisely, S., \& Kroenke, K. (2009). Short-term psychodynamic psychotherapy for somatic disorders. Psychotherapy \& Psychosomatics, 78, 265-274.

Andersen, B., Yang, H., Farrar, W., Golden-Kreutz, D., Emery C., Thornton, L. et al. (2008). Psychologic intervention improves survival for breast cancer patients. Cancer, 113, 3450-3458.

American Psychiatric Association. (2013). Diagnostic and statistical manual of mental disorders (5th ed.). Arlington, VA: American Psychiatric Publishing.

Applebaum, J., Nemets, B., Kaplan, Z., Witztum, E., \& Belmaker, R. (2012). Prevalence of history of childhood sexual abuse in consecutive hospital admissions of women with psychotic diagnosis in Israel: a preliminary report. Psychotherapy \& Psychosomatics, 81, 318-319.

Arnow, B.A. (2004). Relationships between childhood maltreatment, adult health and psychiatric outcomes, and medical utilization. Journal of Clinical Psychiatry, 65, 10-15.

Aspy, C.B., Mold, J.W., Thompson, D.M., Blondell, R.D., Landers, P.S., Reilly, K.E. et al. (2008). Integrating screening and interventions for unhealthy behaviors into primary care practices. American Journal of Preventive Medicine, 35, S373-S380.

Bernini O., Pennato T., Cosci F., \& Berrocal C. (2010). The psychometric properties of the CPAQ in Italian patients with chronic pain. Journal of Health Psychology, 5, 1-10.

Beutel, M., J. Wiltink, J., Till, Y., Wild, P., Münzel, T., Ojeda, F. et al. (2012). Type D personality as a cardiovascular risk marker in the general population: Results from the Gutenberg Health Study. Psychotherapy \& Psychosomatics, 81, 108-117.

Bodenheimer, T., Lorig, K., Holman, H., \& Grumbach, K. (2002). Patient selfmanagement of chronic disease in primary care. JAMA 288, 24692475 .
No es que ciertos trastornos no tengan explicación; es que, en muchos casos, nuestra evaluación es inadecuada, dado que no refleja una perspectiva psicosomática global (Fava \& Sonino, 2009; Sonino \& Peruzzi, 2009).

Desafortunadamente, la mayor parte de los recursos sanitarios se concentra en la atención biomédica. La excesiva importancia concedida a la farmacología ha dado lugar a un peligroso reduccionismo mediante el cual se descuida con negligencia que los resultados terapéuticos son siempre la consecuencia de múltiples variables, específicas o inespecíficas, y de distinta naturaleza (Fava \& Sonino, 2005). En este contexto, el modelo biopsicosocial se presenta no tanto como un marco (estático) conceptual, sino como un "factor" el "factor biopsicosocial"- que podría contribuir con fuerza a estimular la renovación de la asistencia sanitaria (Fava et al., 2012). Ciertamente, dicha renovación es un "reto para la medicina", como ya denunciara Engel (1977) en el subtítulo de su trabajo. No tanto porque en el modelo biopsicosocial se integren variables de distinta naturaleza, sino porque con él se introduce un factor que obstaculiza el mantenimiento de los intereses comerciales y económicos que progresivamente se han establecido en el contexto médico. Así, mientras el reduccionismo biomédico es una de las claves que garantiza el éxito de la propaganda farmacéutica, alimentando con ello el conflicto de intereses en la práctica profesional, el factor biopsicosocial constituye una de sus principales amenazas y, precisamente por ello, todavía hoy se erige como un "reto para la medicina" (Fava et al., 2012).
Bonomi, A., Anderson, M., Rivara, F., Cannon, E., Fishman, P., Carrell, D. et al. (2008). Health care utilization and costs associated with childhood abuse. Journal of General Internal Medicine, 23, 294-299.

Bouman, T.K., \& Visser, S. (1998). Cognitive and behavioral treatment of hypochondriasis. Psychotherapy \& Psychosomatics, 67, 214-221.

Chida, Y., \& Steptoe, A. (2008). Positive psychological well-being and mortality. Psychosomatic Medicine, 70, 741-56.

Clark, D.M., Salkovskis, P.M., Hackmann, A., Wells, A., Fennell, M., Ludgate, J. et al. (1998). Two psychological treatments for hypochondriasis. British Journal of Psychiatry, 173, 218-225.

Cohen, S., Gottlieb, B., \& Underwood, L. (2000). Social relationships and health. En Cohen, S., Underwood, L., \& Gottlieb B. (Eds). Social support measurement and intervention (pp. 3-25). New York: Oxford University Press.

Compas, B., Haaga, D., Keefe, F., Leitenberg, H., \& Williams, D. (1998). Sampling of empirically supported psychological treatments from health psychology: smoking, chronic pain, cancer, and bulimia nervosa. Journal of Consulting and Clinical Psychology, 66, 89-112.

Cosci, F., Pennato, T., Bernini O., \& Berrocal C. (2011). Psychological wellbeing, negative affectivity, and functional impairment in fibromyalgia. Psychotherapy \& Psychosomatics, 80, 256-258.

Daniels, S.R., \& Greer, F.R. (2008). Committee on Nutrition. Lipid screening and cardiovascular health in childhood. Pediatrics, 122, 198-208.

De Fruyt, J., \& Demyttenaere, K. (2009). Quality of life measurement in antidepressant trials. Psychotherapy \& Psychosomatics, 78, 212-219.

Deter, H.C. (2004). Psychosomatic medicine and psychotherapy. Advances in Psychosomatic Medicine, 26, 181-189.

De Vries A.M.M., Forni, V., Voellinger, R., \& Stiefel, F. (2012). Alexithymia in Cancer Patients: Review of the Literature. Psychotherapy and Psychosomatics, 81, 79-86. 
Di Matteo, M.R., Lepper, H.S., \& Croghan, T.W. (2000). Depression is a risk factor for noncompliance with medical treatment. Archives of Internal Medicine, 160, 2101-2107.

Djoussé, L., Driver, J.A., \& Graziano, J.M. (2009). Relation between modifiable lifestyle factors and lifetime risk of heart failure. $J A M A, 302,394$ 400.

Engel, G.L. (1967). The concept of psychosomatic disorder. Journal of Psychosomatic Research, 11, 3-9.

Engel, G.L. (1977). The need for a new medical model: a challenge for biomedicine. Science, 196, 129-136.

Eriksson, M., \& Lindstrom, B. (2006). Antonovsky's sense of coherence scale and the relation with health: a systematic review. Journal of Epidemiology and Community Health, 60, 376-381.

Faravelli, C., Fioravanti, G., Casale, S., Paciello, D., Miraglia, A., Fei, L. et al. (2012). Early life events and gynaecological cancer: A pilot study. Psychotherapy \& Psychosomatics, 81, 56-57.

Fava, G.A. (1999a). Subclinical symptoms in mood disorders: pathophysiological and therapeutic implications. Psychological Medicine, 29, 47-61.

Fava, G.A. (1999b). Well-being Therapy. Psychotherapy \& Psychosomatics, 68, 171-178.

Fava, G.A., Fabbri, S., Sirri, L., \& Wise, T.N. (2007). Psychological factors affecting medical condition: a new proposal for DSM-V. Psychosomatics, $48,103-11$.

Fava, G.A., Guidi, J., Semprini, F., Tomba, E., \& Sonino, N. (2010). Clinical assessment of allostatic load and clinimetric criteria. Psychotherapy and Psychosomatics, 79, 280-284.

Fava, G.A., Ruini, C., Tomba, E., \& Wise, T.N. (2012). The biopsychosocial factor. Psychotherapy \& Psychosomatics, 81, 1-4.

Fava, G.A., \& Sonino, N. (2000). Psychosomatic medicine: emerging trends and perspectives. Psychotherapy \& Psychosomatics, 69, 184-197.

Fava, G.A., \& Sonino N. (2005). The clinical domains of psychosomatics medicine. Journal of Clinical Psychiatry, 66, 849-858.

Fava, G.A., \& Sonino, N. (2009). Psychosomatic assessment. Psychotherapy and Psychosomatics, 78, 333-341.

Fava, G.A., \& Sonino, N. (2010). Psychosomatic Medicine. International Journal of Clinical Practice, 64, 1155-1161

Ferrari, S., Galeazzi, G.M., Mackinnon, A., \& Rigatelli, M. (2008). Frequent attenders in primary care. Psychotherapy and Psychosomatics, 77, 306-314.

Forman, J.P., Stampfer, M.J., \& Curhan, G.C. (2009). Diet and lifestyle risk factors associated with incident hypertension in women. $J A M A, 302$, 401-411

Frasure-Smith, N., \& Lesperance, F. (2003). Depression and other psychological risks following myocardial infarction. Archives of General Psychiatry, 60, 627-636.

Frisina, P.G., Borod, J.C., \& Lepore, S. (2004). A meta-analysis of the effects of written emotional disclosure on the health outcomes of clinical populations. Journal of Nervous and Mental Disease, 192, 629-34.

Gitlin, D.F., Levenson, J.L., \& Lyketsos, C.G. (2004). Psychosomatic medicine: a new psychiatric subspecialty. Academic Psychiatry, 28, 4-11.

Grabe, H., Frommer, J., Ankerhold, A., Ulrich, C., Gröger, R., Franke, G. et al. (2008). Alexithymia and outcome in psychotherapy. Psychotherapy and Psychosomatics, 77, 189-194.

Green, L., Cifuentes, M., Glasgow, R., \& Stange, K. (2008). Redesigning primary care practice to incorporate health behavior change. American Journal of Preventive Medicine, 35, 347-349.

Hafen, B., Jarren, K., Frandsen, K., \& Smith, N. (1996). Mind/Body Health. Boston: Allyn and Bacon.

Halliday, J.L. (1948). Psychosocial medicine. A study of the sick society. London: Heinemann.

Hart, J.T. (1995). Clinical and economic consequences of patients as producers. Journal of Public Health Medicine, 17, 383-386.

Heath, I. (2006). Combating disease mongering: daunting but nonetheless essential. PLoS Medicine, 3, e146.

Hinkle, L. (1967). Human ecology and psychosomatic medicine.Psychosomatic Medicine, 29, 391-395.

Jackson, J.L., de Zee, K., \& Berbano, E. (2004). Can treating depression improve disease outcomes? Annals of Internal Medicine, 140, 1054-1056

Joosten, E.A., De Fuentes-Merillas, L., de Weert, G.H., Sensky, T., van der Staak, C.P., \& de Jong, C. (2008). Systematic review of the effects of shared decision-making on patient satisfaction, treatment adherence and health status. Psychotherapy \& Psychosomatics, 77, 219-226.

Kaplan, R. (2003). The significance of quality of life in health care. Quality of Life Research, 12, 3-16.

Katon, W.J. (2003). Clinical and health services relationships between major depression, depressive symptoms, and general medical illness. Biological Psychiatry, 54, 216-226.

Kaupp, J., Rapaport-Hubschman, N., \& Spiegel, D. (2005). Psychosocial treatments. En: Levenson, J. (ed.). Textbook of Psychosomatic Medicine (pp.923-956). Washington: American Psychiatric Press.

Kissen, D.M. (1963). The significance of syndrome shift and late syndrome association in psychosomatic medicine. Journal of Nervous and Mental Disease, 136, 34-42.

Konnopka A., Schaefert R., Heinrich S., Kaufmann C., Luppa M., Herzog W. et al. (2012). Economics of medically unexplained symptoms: A systematic review of the literature. Psychotherapy \& Psychosomatics, 81, 265275 .

Kroenke, K. (2002). Psychological medicine. BMI, 324, 1536-1537.

Layard, R. (2006). The case of psychological treatment centres. BMJ, 332, 1030-1032.

Leventhal, H., Weinman, J., Leventhal, E.A., \& Phillips, L.A. (2008). Health psychology: the search for pathways between behavior and health. $A n$ nual Review of Psychology, 59, 477-505.

Lipowski, Z.J. (1983). Current trends in consultation-liaison psychiatry. Canadian Journal of Psychiatry, 28, 329-338.

Lipowski, Z.J. (1986). Psychosomatic medicine: past and present. Canadian Journal of Psychiatry, 31, 2-21.

Lishman, W.A. (1998). Organic Psychiatry.Oxford: Blackwell.

Marks, I. (2009). Mental health clinics in the 21st century. Psychotherapy and Psychosomatics, 78, 133-138.

Matarazzo, J.D. (1980). Behavioral health and behavioral medicine: frontiers for a new Health Psychology. American Psychologist, 35, 807-817.

McCauley, J., Kern, D., Kolodner, K., Dill, L., Schroeder, A., DeChant, H. et al. (1997). Clinical characteristics of women with a history of childhood abuse. IAMA, 277, 1362-1368.

McEwen, B.S. (2007). Physiology and neurobiology of stress and adaptation: central role of the brain. Physiological Review, 87, 873-904.

McEwen, B.S., \& Stellar, E. (1993). Stress and the individual. Mechanisms leading to disease. Archives of Internal Medicine, 153, 2093-2101.

Miller, N.E. (1983). Behavioral medicine. Annual Review of Psychology, 34, 131.

Mischel, W., \& Shoda, Y. (1995). A cognitive-affective system theory of personality. Psychological Review, 102, 246-268.

Mitchell, A., Vaze, A., \& Rao, S. (2009). Clinical diagnosis of depression in primary care: a meta-analysis. Lancet, 374, 609-619.

Mokdad, A.H., Marks, J.S., Stroup, D.F., \& Gerberding, J.L. (2004). Actual causes of death in the United States, 2000. JAMA, 291, 1238-1245.

Moorey, S., Cort, E., Kapari, M., Monroe, B., Hansford, P., Mannix, K. et al. (2009). A cluster randomized controlled trial of cognitive behaviour therapy for common mental disorders in patients with advanced cancer. Psychological Medicine, 39, 713-723.

Narayan, K.M., Kanaya, A.M., \& Gregg, E.W. (2003). Lifestyle intervention for the prevention of type 2 diabetes mellitus. Treatments in Endocrinology, 2, 315-320.

Novack D., Cameron O., Epe, E., Ader R., Waldstein S., Levenstein S. et al. (2007). Psychosomatic medicine: the scientific foundation of the biopsychosocial model. Academic Psychiatry, 31, 388-401.

Ogrodniczuk, J.S., Sierra, C., Sochting, I., Joyce, A.S., \& Piper, W.E. (2012). Type D personality and alexithymia among psychiatric outpatients. Psychotherapy \& Psychosomatics, 81, 118-120.

Picardi, A., \& Abeni, D. (2001). Stressful life events and skin disease. Psychotherapy \& Psychosomatics, 70, 118-136.

Porcelli P., Bellomo A., Quartesan R., Altamura M., Iuso S., Ciannameo, I. et al. (2009). Psychosocial functioning in consultation-liaison psychiatry patients. Psychotherapy \& Psychosomatics, 78, 352-358.

Porcelli, P., Domenico, L., Domenico, M., \& Di Masi, A. (2012). Prevalence of allostatic overload syndrome in patients with chronic cardiovascular disease. Psychotherapy and Psychosomatics 81, 375-377.

Porcelli, P., \& Sonino, N. (2007). Psychological Factors Affecting Medical Conditions. A new Classification for DSM-V. Basel: Karger. 
Pressman, S., \& Cohen, S. (2005). Does positive affect influence health? Psychological Bulletin, 131, 925-971.

Prince, M., Patel, V., Saxena, S., Maj, M., Maselko, J., Phillips, M.R. et al. (2007). No health without mental health. Lancet, 370, 859-877.

Romans, S., \& Cohen, M. (2008). Unexplained and underpowered: the relationship between psychosomatic disorders and interpersonal abuse. Harvard Review of Psychiatry, 16, 35-44.

Rose, G. (1985). Sick individuals and sick populations. International Journal of Epidemiology, 14, 32-38.

Roy-Byrne, P.P., Davidson, K.W., Kessler, R.C., Asmundson, G.J.G., Goodwin, R.D., Kubzansky, L. et al. (2008). Anxiety disorders and comorbid medical illness. General Hospistal Psychiatry, 30, 208-225.

Rozanski, A., Blumenthal, J.A., \& Kaplan, J. (1999). Impact of psychological factors on the pathogenesis of cardiovascular disease and implications for therapy. Circulation, 99, 2192-2217.

Ruini, C., \& Fava, G.A. (2013). The polarities of psychological well being and their response to treatment. Terapia Psicologica, 31, 49-57.

Ryff, C. (1989). Happiness is everything, or is it? Explorations on the meaning of psychological well-being. Journal of Personality and Social Psychology, 6, 1069-1081.

Ryff, C., \& Singer, B. (1996). Psychological well-being. Psychotherapy \& Psychosomatics, 65, 14-23.

Ryff, C., \& Singer, B. (1998). The contours of positive human health. Psychological Inquiry, 9, 1-28

Schulz, R., Drayer, R.A., \& Rollman, B.L. (2002). Depression as a risk factor for non-suicide mortality in the elderly. Biological Psychiatry, 52, 205-225.

Schwartz, G.E., \& Weiss, S.M. (1978). Yale conference on behavioral medicine: a proposed definition and statements of goals. Journal of Behavioral Medicine, 1, 3-11.

Seeman, T.E., Singer, B.H., Ryff, C., Dienberg, L., \& Levy-Storms L. (2002). Social relationships, gender, and allostatic load across two age cohorts. Psychosomatic Medicine, 64, 395-406.

Shah, S.U., White, A., White, S., \& Littler, W.A. (2004). Heart and mind: (1) relationship between cardiovascular and psychiatric conditions. Postgraduate Medical Journal, 80, 683-689.

Sharpe, L., \& Schrieber, L. (2012). A blind randomized controlled trial of cognitive versus behavioral versus cognitive-behavioral therapy for patients with rheumatoid arthritis. Psychotherapy \& Psychosomatics, 81, 145152.

Shonkoff, J.P., Boyce, W.T., \& McEwen, B.S. (2009). Neuroscience, molecular biology and the childhood roots of health disparities. JAMA, 301, 2252-2259.

Sirri, L., \& Fava, G.A. (2013). Diagnostic criteria for psychosomatic research and somatic symptom disorders. International Review of Psychiatry, 25, 1930 .
Sirri, L., Fava, G.A., \& Sonino, N. (2013). The unifying concept of illness behavior. Psychotherapy and Psychosomatics, 82, 74-81.

Sirri, L., Grandi, S., \& Fava, G.A. (2008). The illness attitude scales. Psychotherapy and Psychosomatics, 77, 337-350.

Sonino, N., \& Fava, G.A. (2007). Rehabilitation in endocrine patients: a novel psychosomatic approach. Psychotherapy and Psychosomatics, 76, 319324.

Sonino, N., Tomba, E., \& Fava, G.A. (2007). Psychosocial approach to endocrine disease. Advances in Psychosomatic Medicine, 28, 21-33.

Sonino, N., \& Peruzzi, P. (2009). A psychoneuroendocrinology service. Psychotherapy \& Psychosomatics, 78, 346-351.

Stone, N.J. (2004). Focus on lifestyle change and the metabolic syndrome. Endocrinology and Metabolism Clinics of North America, 33, 493-508.

Taylor, G.J., \& Bagby R. (2004). New trends in alexithymia research. Psychotherapy and Psychosomatics, 73, 68-77.

Testa, M., \& Simonson, D. (1996). Assessment of quality of life outcomes. New England Journal of Medicine, 334, 835-840.

Tomba, E., \& Bech, P. (2012). Clinimetrics and clinical psychometrics: Macro- and micro-analysis. Psychotherapy \& Psychosomatics, 81, 333-343.

Tomba, E., \& Offidani, E. (2012). A clinimetric evaluation of allostatic overload in the general population. Psychotherapy \& Psychosomatics, 81, 378379.

Turk, D.C., Swanson, K.S., \& Tunks, E.R. (2008). Psychological approaches in the treatment of chronic pain patients. Canadian Journal of Psychiatry, 53, 213-223

Vedsted, P., \& Christensen, M.B. (2005). Frequent attenders in general practice care: a literature review with special reference to methodological considerations. Public Health, 119, 118-137.

Venditti, F. Cosci, F., Bernini, O., Berrocal C. (2013). Criterion validity of the Diagnostic Criteria for Psychosomatic Research in patients with morbid obesity. Psychotherapy \& Psychosomatics, 82, 411-412.

Walker, E., Gelfand, A., Katon, W., Koss, M., Korff, M., Bernstein, D. et al. (1999). Adult health status of women with histories of childhood abuse and neglect. American Journal of Medicine, 107, 332-339.

Wise, T.N. (2000). Consultation liaison psychiatry and psychosomatics: strange bedfellows. Psychotherapy \& Psychosomatics, 69, 181-183.

World Health Organization (1948). World Health Organization Constitution. Geneva: WHO.

Wright, R., Rodriguez, M., \& Cohen, S. (1998). Review of psychosocial stress and asthma. Thorax, 53,1066-1074.

(Artículo recibido: 06-02-2015; revisado: 19-07-2015; aceptado: 10-10-2015) 